\title{
Fast Solar Radiation Pressure Modelling with Ray Tracing and Multiple reflections
}

\author{
Zhen Li $^{\mathrm{a}, *}$, Marek Ziebart ${ }^{\mathrm{a}}$, Santosh Bhattarai ${ }^{\mathrm{a}}$, David Harrison ${ }^{\mathrm{a}}$, Stuart Grey ${ }^{\mathrm{b}}$ \\ ${ }^{a}$ University College London, Gower Street, London WC1E 6BT, United Kingdom \\ ${ }^{b}$ University of Strathclyde, 16 Richmond Street, Glasgow G11XQ, United Kingdom
}

\begin{abstract}
Physics based SRP (Solar Radiation Pressure) models using ray tracing methods are powerful tools when modelling the forces on complex real world space vehicles. Currently high resolution $(1 \mathrm{~mm})$ ray tracing with secondary intersections is done on high performance computers at UCL (University College London). This study introduces the BVH (Bounding Volume Hierarchy) into the ray tracing approach for physics based SRP modelling and makes it possible to run high resolution analysis on personal computers. The ray tracer is both general and efficient enough to cope with the complex shape of satellites and multiple reflections (three or more, with no upper limit). In this study, the traditional ray tracing technique is introduced in the first place and then the BVH is integrated into the ray tracing. Four aspects of the ray tracer were tested for investigating the performance including runtime, accuracy, the effects of multiple reflections and the effects of pixel array resolution.Test results in runtime on GPS IIR and Galileo IOV (In Orbit Validation) satellites show that the BVH can make the force model computation 30-50 times faster. The ray tracer has an absolute accuracy of several nanonewtons by comparing the test results for spheres and planes with the analytical computations. The multiple reflection effects are investigated both in the intersection number and acceleration on GPS IIR, Galileo IOV and Sentinel-1 spacecraft. Considering the number of intersections, the 3rd reflection can capture $99.12 \%, 99.14 \%$, and $91.34 \%$ of the total reflections for GPS IIR, Galileo IOV satellite bus and the Sentinel-1 spacecraft respectively. In terms of the multiple reflection effects on the acceleration, the secondary reflection effect for Galileo IOV satellite and Sentinel-1 can reach 0.2 $\mathrm{nm} / \mathrm{s}^{2}$ and $0.4 \mathrm{~nm} / \mathrm{s}^{2}$ respectively. The error percentage in the accelerations magnitude results show that the 3rd reflection should be considered in order to make it less than $0.035 \%$. The pixel array resolution tests show that the dimensions of the components have to be considered when choosing the spacing of the pixel in order not to miss some components of the satellite in ray tracing. This paper presents the first systematic and quantitative study of the secondary and higher order intersection effects. It shows conclusively the effect is non-negligible for certain classes of misson.
\end{abstract}

Keywords: SRP modelling; Fast Ray Tracing; Multiple-reflection; GPS IIR; Galileo IOV; Sentinel-1

\section{Introduction}

SRP ( Solar radiation pressure) has effects on all the artificial satellites. It is the largest non-gravitational force for high altitude satellites. Take GNSS ( Global Navigation Satellite System) satellites as an example, SRP force will make them drift several hundred meters in one day (Springer et al, 1999; Montenbruck and Gill, 2005; Ziebart et al, 2005). Thus it is very important to accurately model SRP for the sake of orbit quality.

Currently, the strategies for SRP modelling presented in the precise orbit determination community may be broadly divided into two categories. The first category is the empirical approach. Triangular functions like sine and cosine are used in the along track, radial and cross track to absorb the un-modelled non-gravitational forces. This method has been applied to the orbit determination of many missions such as Sentinel-1 (Peter et al, 2017), Jason (Cerri et al, 2010), HY-2A (Gao et al,

\footnotetext{
${ }^{*}$ Corresponding author

Email addresses: hpulizhen@163.com (Zhen Li),

m.ziebart@ucl.ac.uk (Marek Ziebart), s.bhattarai@ucl.ac.uk (Santosh Bhattarai), david.harrison.14@ucl.ac.uk (David Harrison), stuart.grey@strath.ac.uk (Stuart Grey)
}

2015), GRACE (Gravity Recovery and Climate Experiment) (Kang et al, 2006), GOCE (Gravity field and steady-state Ocean Circulation Explorer) (Casotto et al, 2013) and GNSS satellites (Sibthorpe et al, 2011; Arnold et al, 2015). Although this approach has been widely used by the community, it does have drawbacks. One aspect is that in the estimation of empirical parameters, there is a risk of mixing orbit dynamic parameters with other physical parameters such as earth orientation parameters and geocentre (Meindl et al, 2013). The other aspect is that the parameters in the empirical model have no clear physical meaning which means it is less helpful for understanding what really happened to the satellites in space.

The second category is to develop high-fidelity SRP model that considers the interactions between solar rays and satellite surfaces. This approach is very convenient for analysing the effects of different physical parameters on the orbit. In addition, in the data processing of precise orbit determination, the highfidelity model can also help to reduce the correlations between estimates which means a tight constraint is introduced on these parameters. In practice, the tight constraint can improve the estimated parameters (Ziebart, 2004; Solano, 2014; Cerri et al, 2010). 
However, the complex shape and multiple materials of the satellite surfaces have to be considered in developing the highfidelity SRP model. One way to deal with this problem is by using the ray tracing technique. For satellites that have dynamic components such as the solar panels of GNSS satellites, the calculation of the dynamic components are separated for the efficiency of ray tracing though this may cause ignoring of shadowing effect from solar panels to the satellite bus. For the satellites that with all the components fixed such as Sentinel-1, the ray tracing can be applied directly.

The ray tracing technique accounts for the interactions between each ray and satellite surfaces. But the number of rays can reach over $2.5 \times 10^{7}$ for satellites with a size of $5 \times 5 \mathrm{~m}^{2}$ at only one direction of solar flux. Therefore, it is necessary to find a way to make this ray tracer more efficient in computation.

Generally, this problem can be solved from two aspects. One aspect is to reduce the number of rays that needs to be calculated. The other aspect is to speed up the process of finding the intersections between rays and the spacecraft surfaces. Thus two questions will be produced with respect to the above two aspects. 1) How is the spacecraft geometry represented? 2) How to find the intersections as quick as possible? Regarding the first question, the satellite bus is represented by simple geometries that can be described by mathematical equations. The intersections can be calculated in an analytical way with this representation. As for the second question, there exits the first attempt to speed up the modelling computation by using block modelling approach (Sibthorpe, 2006). In this study, the BVH (Bounding Volume Hierarchy) data structure is used in organising the components of the satellite bus. The essence of the BVH is a binary tree (or K-Dimensional Tree ) that stores primitives in the scene where each node represents a bounding volume (Lauterbach et al, 2009; Bittner et al, 2015).

This paper firstly introduces the physical basis of SRP and is followed by the general ray tracing approach for SRP modelling. The representation of the satellite bus geometry and the intersection algorithm for each geometry are described along with the implementation of the ray tracer. Secondly, the BVH data structure is applied to the ray tracer to improve the performance. This BVH data structure reorganises the components of the satellite bus and speeds up the process of searching for the possible intersection components. Finally, the performance of the ray tracer is tested in four aspects, including absolute accuracy, runtime, the effects of multiple reflections and the effects of pixel array resolution.

\section{The physics of radiation pressure}

The physical foundation is the basis for solar radiation pressure modelling. The theory of radiation pressure was revealed by Maxwell as an assertion based on his theory of electromagnetism. The radiation pressure phenomenon was then validated in experiments by Nichols, Pyotr Nicholaievich Lebedev and Hull (Lebedev, 1901; Nichols and Hull, 1902, 1903). According to Maxwell's theory, an electromagnetic wave carries momentum which can be transferred to a reflecting or absorbing surface hit by the wave. The momentum change will generate a force on the surface. By applying Einstein's special theory of relativity and the theorem of impulse, the force generated by the electromagnetic wave is obtained. For a photon of frequency $f$, the momentum $p$ is given

$$
p=\frac{h f}{c}
$$

where $h$ is Planck's constant.

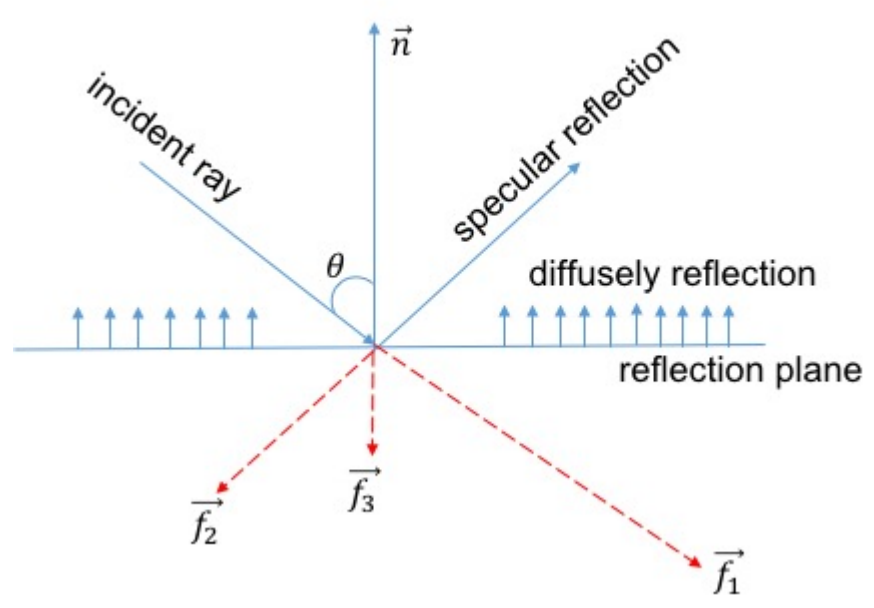

Figure 1: This figure shows the force effects due to the incident ray on a plane

Figure 1 shows the process of reflection that happened on a plane. Assume there are $N_{f}$ photons at frequency $f$ in the incident ray going through unit area at unit time $d t, v_{f} N_{f}$ photons are reflected. Within the reflected photons, there are $\mu_{f} v_{f} N_{f}$ photons getting specularly reflected and $v_{f}\left(1-\mu_{f}\right) N_{f}$ photons getting diffusely reflected. For the diffuse reflection, assume the surface meets the requirement of Lambert assumption which means the intensity of the ray falls off by a factor of $\cos \theta$ away from the normal to the surface. The proportion of diffusely reflected radiation that is emitted normal to surface is $\frac{2}{3}$ (Ziebart, 2004; Fliegel et al, 1992). Applying the conservation of momentum to the radiation flux and the surface:

$$
\frac{N_{f} h f}{c} \mathbf{s}=\mu_{f} v_{f} \frac{N_{f} h f}{c} \mathbf{r}+\frac{2}{3} v_{f}\left(1-\mu_{f}\right) \frac{N_{f} h f}{c} \mathbf{n}+\Delta \mathbf{p}_{f}
$$

where

$\mathbf{s}$ is the unit vector of incident ray.

$\mathbf{r}$ is the unit vector of specularly reflected direction.

$\mathbf{n}$ is the unit normal vector of the surface.

$\Delta \mathbf{p}_{f}$ is the momentum change due to the hit of photons of frequency $f$ per unit time.

Integrating over all the solar radiation spectrum ( assume the wavelength range is from 0 to $\infty$ ), the momentum change can be expressed:

$$
\Delta \mathbf{p}=\int_{0}^{\infty} \Delta \mathbf{p}_{f} d f=\int_{0}^{\infty}\left(\mathbf{s}-\mu_{f} v_{f} \mathbf{r}-\frac{2}{3} v_{f}\left(1-\mu_{f}\right) \mathbf{n}\right) \frac{N_{f} h f}{c} d f
$$


According to the theorem of impulse, the changes in momentum per unit time is actually the force.

$$
\mathbf{F}=\Delta \mathbf{p}
$$

Given the power density $W$ ( photon flux multiply the energy per photon) in units of Watt $\cdot \mathrm{m}^{-2}$, the reflectivity $v$ and specularity $\mu$ over the radiation spectrum, the area of the surface $A$ and the incident angle $\theta$. The power of the radiation that reaches the surface is $W A \cos \theta$. Therefore

$$
\mathbf{F}=\frac{W A \cos \theta}{c}\left(\mathbf{s}-v \mu \mathbf{r}-\frac{2}{3} v(1-\mu) \mathbf{n}\right)
$$

where the specularly reflected direction can be expressed

$$
\mathbf{r}=\mathbf{s}-2(\mathbf{n} \cdot \mathbf{s}) \mathbf{n}
$$

and

$$
\cos \theta=-\mathbf{s} \cdot \mathbf{n}
$$

Equation (5) is the foundation of all the solar radiation pressure calculation. Fliegel et al (1992) and Ziebart (2004) also gave out the analytical formula of direct solar radiation pressure in normal and shear components.

\section{Spacecraft geometry representation and intersections}

Usually the real world satellites have very complex shapes. However in the modelling of the satellite geometry, some simplifications and assumptions have to be made. Regarding the description of the satellite bus geometry, one way is to use a tessellation approach which represents complex geometry with a model consisting only of planar triangles (Grey et al, 2017). However, this tessellation approach will introduce errors for the curved surfaces such as spheres, cylinders, parabolic dishes and so on. Therefore this study represents the real world satellite bus with components of simple geometries (Ziebart, 2001, 2004). Such as polygons, discs, spheres, cylinders, cones and so on. These simple geometries can be described by rigorous mathematical equations and the intersections can be accurately computed.

In order to apply the BVH in ray tracing, all the components have to be surrounded by volumes, this can help to speed up the intersection searching process. In this study, the AABB (Axis Aligned Bounding Box) is used. The orientation of AABB is aligned to the three axes of the satellite BFS (Body Fixed System ). The AABB for every component is stored as the minimum and maximum coordinates in $\mathrm{x}, \mathrm{y}$ and $\mathrm{z}$. For every component, it also needs a Local Body System (LBS) to describe the geometry. The transformation matrix between the local body coordinate system and the satellite body fixed frame will be computed when building the geometry model.

The intersections between these geometries and the ray can be obtained by solving the simultaneous equations composed by the equation of ray and the equation of the geometry. Usually the ray is expressed in the BFS with a start point $\mathbf{a}$ and a unit direction vector $\mathbf{d}$.

$$
\mathbf{s}=\mathbf{a}+t \mathbf{d}
$$

where $t$ is the length of the ray.

The first step is to convert the ray from BFS to LBS. Once the intersection is computed and confirmed, it needs to be converted back to the BFS. The following section will discuss the solutions to the intersection problems for all the geometries.

\section{Planar polygon $\&$ circle $\&$ ring}

The intersection calculation for planar geometries requires two steps. The first step is to get the candidate intersection which is the intersection between the ray and the plane. The next step is to check if the candidate intersection is inside the shape. If the candidate intersection is outside the shape, it will not be accepted as an intersection. In the local body coordinate system, the equation of a plane is

$$
\mathbf{n}^{\mathrm{T}} \mathbf{x}=0
$$

where $\mathbf{n}$ is the normal vector of the plane, $\mathbf{x}$ is the coordinates of intersection point. Substituting the ray equation into this equation, the parameter $t$ in the ray equation can be solved:

$$
t=-\frac{\mathbf{n}^{\mathrm{T}} \mathbf{a}}{\mathbf{n}^{\mathrm{T}} \mathbf{d}}
$$

Which means the distance between the start point of the ray and the candiate intersection is determined, thus the candidate intersection point can be obtained. Once the candidate intersection is computed, the next step is to check if this candidate is inside the polygon or circle or the ring. This process will be different for the different geometries. For the polygons, this will involve the winding number theory in topology. The winding number of a closed curve in the plane around a given point is an integer representing the total number of times that curve travels counter clockwise around the point. If the winding number of the polygon for the candidate intersection is 0 , the candidate is outside the polygon otherwise it is inside the polygon (Hormann and Agathos, 2001). For circles, one only needs to check the distance between the centre of the circle and the candidate intersection. If the distance is less than the radius of the circle, then the candidate intersection gets accepted. It is similar for the planar ring, if the distance is greater than the radius of inner circle and less than the radius of external circle, the candidate gets accepted.

\section{Sphere}

In the local body frame of a sphere, the equation of a sphere can be described by

$$
\mathbf{x}^{T} \mathbf{x}=R^{2}
$$

where $R$ is the radius of the sphere.

Substituting the equation of the ray into this equation, a quadratic with respect to parameter $t$ is obtained

$$
\mathbf{d}^{T} \mathbf{d} t^{2}+2 \mathbf{d}^{T} \mathbf{a} t+\mathbf{a}^{T} \mathbf{a}-R^{2}=0
$$

If the quadratic has two solutions, the smaller solution will be chosen as the intersection. Because here parameter $t$ represents 


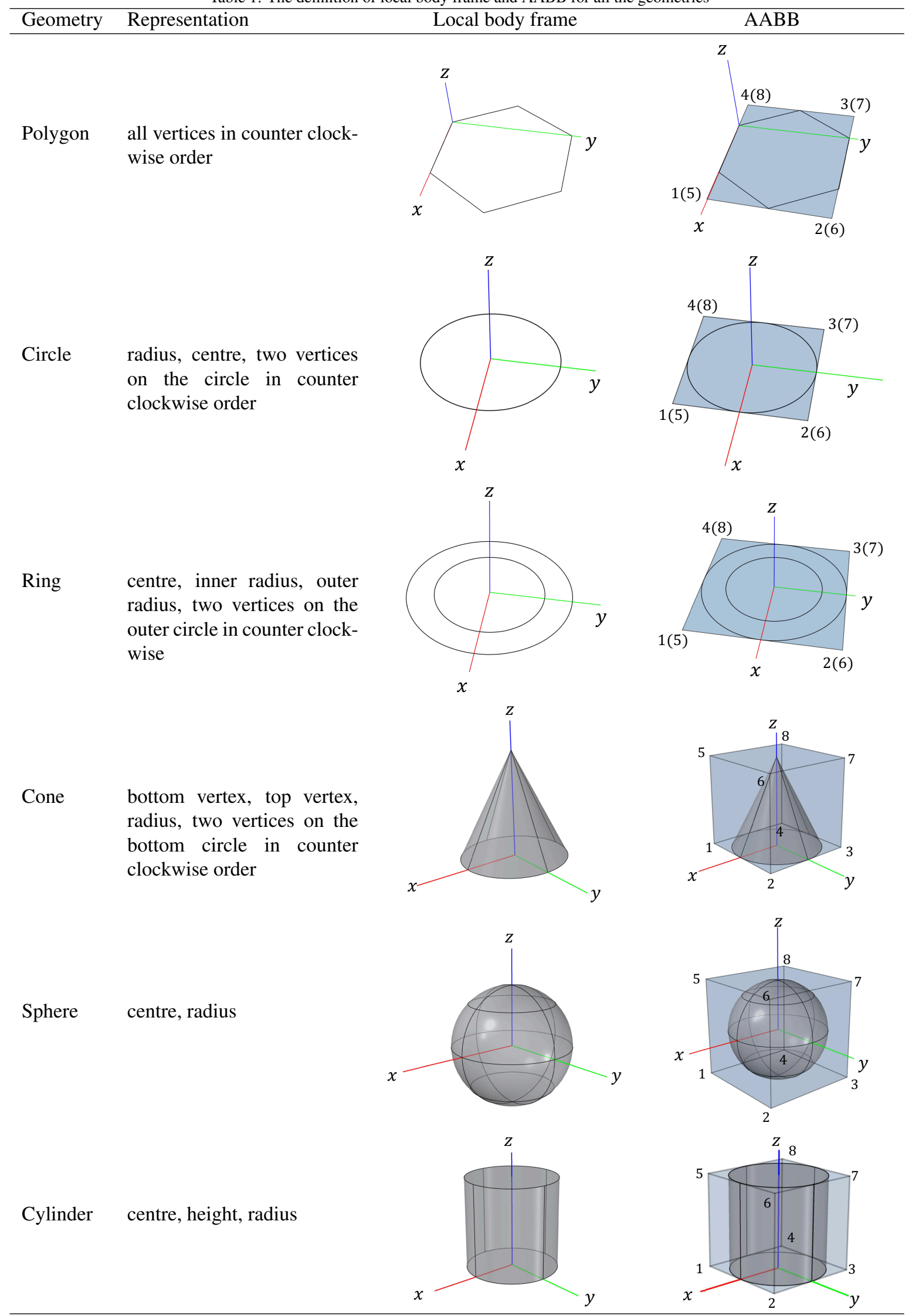


the distance from the start of ray to the intersection, the smaller $t$ means the first intersection of the ray. If the quadratic has only one solution, that means the ray is exactly tangent to the sphere.

\section{Cylinder}

The equation of a cylinder is

$$
\left\{\begin{array}{l}
x^{2}+y^{2}=R^{2} \\
0 \leq z \leq h
\end{array}\right.
$$

where $R$ is the radius of the cylinder and $h$ is the height of the cylinder, i.e. the distance from centre of the bottom to centre of the top.

This set of equations does not include the bottom and top circles. In this study, the cylinder is open capped. Expanding the equation of ray into scalar form and combining them together, a quadratic with respect to parameter $t$ is obtained:

$$
A t^{2}+B t+C=0
$$

where $A=x_{d}^{2}+y_{d}^{2}, B=2\left(x_{d} x_{a}+y_{d} y_{a}\right), C=x_{a}^{2}+y_{a}^{2}-R^{2}$. It is necessary to check the $\mathrm{z}$ component of the solution and make it meet the condition $0 \leq z \leq h$. If the above quadratic has two solutions, the smaller one would be the solution.

\section{Cone}

The equation of a conical surface is

$$
\left\{\begin{array}{l}
z-h=-\frac{h}{R} \sqrt{x^{2}+y^{2}} \\
0 \leq z \leq h
\end{array}\right.
$$

where $R$ is the radius of the bottom circle, $h$ is the height of the cone. Combining this equation with the equation of the ray, it will generate a quadratic with respect to parameter $t$

$$
A t^{2}+B t+C=0
$$

where $A=z_{d}^{2}-\frac{h^{2}}{R^{2}}\left(y_{d}^{2}+x_{d}^{2}\right), B=2\left(z_{d} z_{a}-h z_{d}-\frac{h^{2}}{R^{2}}\left(x_{d} x_{a}+y_{d} y_{a}\right)\right)$, $C=z_{a}^{2}+h^{2}-2 h z_{a}^{2}-\frac{h^{2}}{R^{2}}\left(x_{a}^{2}+y_{a}^{2}\right)$.

Check all the solutions to make sure the $\mathrm{z}$ coordinate of intersection meets the second equation of conical surface, i.e. $0 \leq z \leq h$. If the condition has been met, then a smaller will be chosen as the solution.

\section{Traditional ray tracing for SRP modelling}

The ray tracing approach can capture small components of the satellite geometry and it is easy to deal with complex shapes. In the modelling of solar radiation pressure, a pixel array is generated with each pixel as the start point of the rays. The pixel array is placed in the direction of the sun with some distance away from the spacecraft (Ziebart, 2001, 2004; Ziebart et al, 2005; Tan et al, 2016). In practice, the distance is set to make sure that the pixel array is outside of the spacecraft. The normal to the pixel array is pointing to the origin of the body-fixed frame of the spacecraft. All the rays are parallel and they are all in the normal direction of the pixel array. This is under the assumption that the sun is so far away from the earth orbit spacecraft that all the rays are assumed to be parallel. Generally, the size of the pixel array is determined based on the size of the spacecraft, the requirement for this is that the pixel array has to fully cover the whole spacecraft, otherwise some rays may be missing. The general process of ray tracing includes three parts: 1) Input the satellite geometry description file. 2) Set up the pixel array. 3) For every ray starting from the pixel array, search all the geometry components to check the intersection. If there is an intersection for the ray, calculate the radiation force and then sum up. This intersection function will be called recursively to deal with the multiple reflection of the ray.

\subsection{Construct the geometry model of the spacecraft}

After obtaining the spacecraft information, the information is reorganized and expressed as the combination of basic geometries described in table1. All the geometry information is written into a description file firstly. The content in the description file includes not only the geometry information but also the optical properties such as reflectivity and specularity of each component. For the convenience of implementation in intersection check, there are two operations need to be done in the process of constructing the spacecraft geometry. The first is to establish the local body frame for every component in the description file according to the definition in table1. The actual implementation about establishing the local body frame is to store the transformation parameters between the local body frame and the spacecraft body fixed frame. The transformation parameters include the coordinate of origin of the local body frame in spacecraft body fixed frame and the three unit vectors of the local body frame axis in spacecraft body fixed frame. The second is to build an AABB (Axis Aligned Bounding Box) for every component both in local body system and spacecraft body fixed frame. This AABB has two functions, one is help to determine the size of the pixel array, the other is to help speed up the intersection process using BVH algorithm.

\subsection{Set up the pixel array}

In the modelling of the solar radiation pressure, every direction of the solar radiation is simulated by a pixel array. The pixel array is placed at $d_{0}=100$ meters away from the spacecraft in calculation. The main task for setting up the pixel array includes two aspects, the first is to determine the size of the pixel array and the second is to construct Pixel Array Coordinate system (PAC) that connects the pixel and the components in spacecraft body fixed frame. In the spacecraft body fixed frame, the direction of pixel array can be represented by a longitude and a latitude as shown in Fig. 2. The definition of PAC system is described as follows:

- 1) Rotate $X$ axis around $Z$ axis with angle $\lambda$ in counter clockwise order. Make sure that $\mathrm{X}$ axis of BFS lies in the projection of satellite-sun vector in XOY plane of BFS.

- 2) Rotate $Z$ axis around $Y$ axis with angle $\frac{3 \pi}{2}-\varphi$ in counter clockwise order so that $\mathrm{Z}$ is pointing from the sun to the spacecraft. 
- 3) Move the origin of BFS to the position of the sun.

Therefore the transformation between these two coordinate system is:

$$
\mathbf{x}_{P A C}=\mathbf{R} \cdot \mathbf{x}_{B F S}+\mathbf{x}_{0}
$$

where

$$
R=\left(\begin{array}{ccc}
-\sin \varphi \cos \lambda & -\sin \varphi \sin \lambda & \cos \lambda \\
-\sin \lambda & \cos \lambda & 0 \\
-\cos \varphi \cos \lambda & -\cos \varphi \sin \lambda & -\sin \varphi
\end{array}\right)
$$

and

$$
\mathbf{x}_{0}=\left(\begin{array}{lll}
-d_{0} \cos \varphi \cos \lambda & -d_{0} \cos \varphi \sin \lambda \quad-d_{0} \sin \lambda
\end{array}\right)^{T}
$$

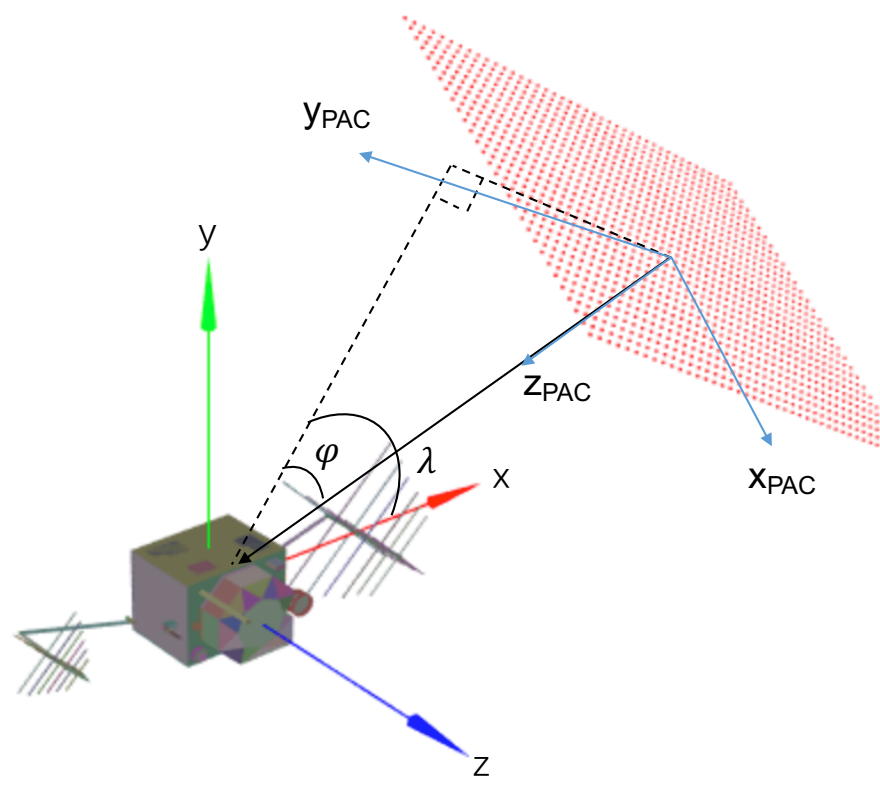

Figure 2: This figure shows the definition of the Pixel Coordinate System (PAC) and the representation of latitude $\varphi$ and longitude $\lambda$ of the sun in spacecraft BFS

Once the Pixel Array Coordinate system (PAC) is established, the size of the pixel array is calculated by converting the AABB of the whole spacecraft into PAC system, then the ranges of $\mathrm{x}$ and $\mathrm{y}$ coordinates in the PAC system are found. Because the pixel array is aligned along the $\mathrm{x}$ and $\mathrm{y}$ axis of PAC, thus the length of the pixel array in $\mathrm{x}$ and $\mathrm{y}$ direction are computed according to the distance between the maximum and the minimum coordinates of the AABB respectively. The number of the pixels in each direction is calculated with the length divided by the spacing of the pixel array.

\subsection{Intersections check and force calculation}

For every ray emitted from the pixel array, it needs a loop to search all the components of the spacecraft to check the intersections. The intersection algorithm for individual component is described in section 3. The ray may intersect multiple components, but only the closest intersection to the pixel is accepted as the real intersection. This is done by checking the distance from the pixel to the candidate intersection. Once the intersection is confirmed, the optical properties of the component and the normal to the surface at the intersection are also recorded. The force due to each ray is computed according to equation (5). If the pixel is small enough, the curved surface could be treated as a differential plane $A$. The relation between $A$ and the area of the pixel $A_{0}$ is:

$$
A_{0}=A \cos \theta
$$

$A_{0}$ is computed according to the spacing of the pixel array. Thus in equation (5), term $A \cos \theta$ should be replaced by $A_{0}$ for every intersected ray.

In the ray tracing, the Total Solar Irradiance (TSI) is set to be $1368 \mathrm{~W} \cdot \mathrm{m}^{-2}$ (Hastings and Garrett, 2004). TSI is actually varying with time and has a characteristics of the solar cycle. The variation should have impacts to the SRP modelling as TSI is an input to the ray tracing approach. However, we only focus on the ray tracing approach itself and assume all the inputs are correct and accurate as they should be.

The intensity of the ray is initially set to be $100 \%$ of the TSI. As for the multiple reflections, only the specular reflection is considered, thus the intensity of the reflected ray has to be reduced by $\mu \nu$ of the last intersected component (Ziebart, 2001, 2004; Ziebart et al, 2005). Ignoring the effects of diffusely reflected radiation will introduce some mismodelling, resultant force caused by the secondary intersections will effectively be negligible (Ziebart, 2001) because of the tendency for diffuse rays from many directions to cancel each other' effects. In addition, a similarly rigorous approach for considering the effects of the diffuse contribution in the multiple reflections would be impractical, as it would end up with exponential growth in the computation.

For the multiple specular reflections, the start point of the secondary reflected ray is the last intersection and the direction is the specularly reflected direction. After setting up the reflected rays, the intersection process is called recursively till it strikes the maximum number of reflection.

\section{Application of BVH in SRP modelling}

BVH ( Bounding Volume Hierarchy) is a tree data structure on a set of geometric primitives, it has been widely used in collision detection and ray tracing (Lauterbach et al, 2009; Günther et al, 2007; Sopin et al, 2011; Bittner et al, 2015). The essence of $\mathrm{BVH}$ is to organise the geometric objects that are wrapped in bounding volume in a binary tree. It recursively partitions the bounding volume by planes and stores the children in the binary tree.

The application of BVH includes the construction of the $\mathrm{BVH}$ tree and traverse of the tree. The details of building the $\mathrm{BVH}$ with the spacecraft geometries and searching for the intersections are discussed in the following sections.

\subsection{Construction of $B V H$ for the spacecraft}

Since all the components of the satellite geometry are surrounded by $\mathrm{AABBs}$, the $\mathrm{AABB}$ for the whole spacecraft is easily found by grouping the AABBs of all the components. The 
root node of the BVH is the spacecraft AABB. The establishment of the $\mathrm{BVH}$ tree is the process of partitioning the $\mathrm{AABB}$ into different levels of sub-AABB and store them into a binary tree. For each AABB, the geometric centre is chosen as its reference point. The key factor that affects the efficiency of a BVH is how to choose the partition plane. There are many algorithms of choosing the partition plane in an optimized way (Sopin et al, 2011). Because how to improve the efficiency of BVH in ray tracing is not the focus of this study, a widely used approach is applied in this study. This approach is in two steps. The first step is to choose the longest axis (this axis can either be $\mathrm{x}, \mathrm{y}$ or $\mathrm{z}$ axis) of the AABB. Step two is to choose the partition plane and make it go through the middle point of the longest axis. For every partition, the AABB is divided into two sub-volumes. The steps of building the BVH are as follows:

- 1) Let the root node be the AABB of the whole spacecraft geometry.

- 2) Choose the partition plane of the root node.

- 3) Loop over all the geometry components, if the reference point of the AABB for the geometry lies on the left side of the partition plane, the component will be stored in the left child, otherwise the component will be sent to the right child.

- 4) Repeat step1 to step3 for both the left and right child till the number of geometry of the children is 1 . The nodes that have only one component inside are treated as leaves.

\subsection{Traverse and intersection check}

The traverse of the BVH tree is the key part of searching the intersection. Because all the nodes of the $\mathrm{BVH}$ tree are surrounded by $\mathrm{AABBs}$, if the ray misses the $\mathrm{AABB}$, the ray will not hit any components inside the $\mathrm{AABB}$. If the ray hits the AABB of that node, all the children of that node should be checked until it reaches the leaves of the tree. For the leaves of the tree, because they are one of the simple geometries described in the previous sections, the intersection algorithm for them can be applied. The closest intersection to the start of the ray is the final solution. The steps for traversing the BVH tree are as follows:

- 1) Start from the root of the BVH tree, if the ray hits the AABB, then check the left child and the right child. Otherwise, the ray will not hit any part of the spacecraft.

- 2) Repeat the above step till it reaches the leaves. The intersection algorithm for the leaves will be executed If the ray hits the leaf geometry, the distance from this intersection to the start of the ray is record.

- 3) Finally, the real intersection is the one that is closest to the start of the ray.

Similarly, this traverse algorithm is also designed to be recursive. This traverse algorithm will return false if there is no intersection, otherwise it will return true. The optical properties, normal vector to the intersected surface and coordinates of intersection will be returned as well. In this traverse process, one key part is to check the intersection of a ray and the bounding box. This has to be ran for so many times that it is very important to make it fast. This study applied an efficient and robust ray-box intersection algorithm (Williams et al, 2003) .

\section{Test and validation}

In this section, the processes for verifying the input (i.e. the spacecraft geometrical model) and testing the performance of the algorithm are described. Quality control checks are carried out to verify the geometry and to confirm that the ray-tracer detects the spacecraft in a correct way. To assess the performance of the ray tracer, a number of tests were done to investigate various aspects. These include tests to examine the absolute accuracy of the force computations and tests to determine the impact of pixel-array spacing on the accuracy of the result. Also, the impact of introducing $\mathrm{BVH}$ into the ray-tracing method is analysed using runtime (with and without $\mathrm{BVH}$ ) comparison tests. Finally, the impact of neglecting the effects of reflected radiation, considering up to 10 reflections, are assessed using models for the GPS IIR and Galileo IOV satellite bus, and a model for the Sentinel-1 spacecraft, as shown in Fig. 3.

The spacecraft models are produced using data from a variety of sources (e.g. design drawings, CAD models). This data is used to represent the surface geometry of the spacecraft using the geometric primitives described in Table 1. The information on the spacecraft models is shown in Table 2. The mass of the satellites may be varying depends on different satellites and operation state. The information of GPS IIR satellite can be found from Ziebart et al (2003). For Galileo IOV satellite, we use the data published at European GNSS Service Centre (European Global Navigation Satellite Systems Agency, 2017). The mass of Sentinel-1 satellite is obtained from Peter et al (2017).

Table 2: The information on the spacecraft model

\begin{tabular}{llll}
\hline Items & GPS IIR & Galileo IOV & Sentinel-1 \\
\hline mass & $1086.451 \mathrm{~kg}$ & $696.815 \mathrm{~kg}$ & $2158.777 \mathrm{~kg}$ \\
$\#$ of compo- & 182 & 590 & 4081 \\
nents & & & \\
min. dimen- & $1.27 \mathrm{~cm}$ & $5.0 \mathrm{~cm}$ & $2.2 \mathrm{~cm}$ \\
sion & & & \\
\hline
\end{tabular}



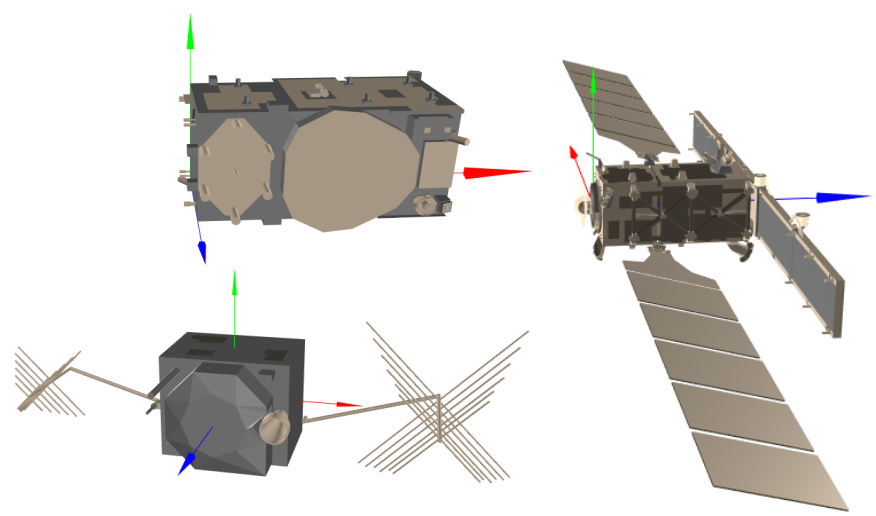

Figure 3: Visualisation of the spacecraft models used in this study: Galileo IOV bus (upper-left), GPS IIR bus (lower-left) and Sentinel-1 (right). For each model, the coordinate systems shown represent the spacecraft BFS $x$ (red), y (green) and $\mathrm{z}$ (blue) axes.

\subsection{Quality control checks of the spacecraft models}

Two simple quality control (QC) checks are used to verify the spacecraft models. First, the spacecraft model file is examined in a $3 \mathrm{D}$-viewer, where it is possible to rotate around the spacecraft model to assess the data for errors in the geometry. The models for the Galileo IOV bus, GPS IIR bus and Sentinel1 spacecraft, as seen in the spacecraft model viewer are shown in Fig. 3. The second QC check is a coarse intersection test that is used to confirm that the ray-tracing software is correctly detecting the spacecraft surface. In Fig. 4, the results of two coarse intersection tests on the Galileo IOV bus model, at pixel array spacings of $1 \mathrm{dm}$ (left) and $1 \mathrm{~cm}$ (right), are shown. In the $3 \mathrm{D}$-viewer, intersections are shown as red dots. With the $1 \mathrm{~cm}$ intersection test, it is possible to show the effectiveness of the ray-tracing software in capturing the effects of self-shadowing - note the shadows cast by the cylinders of the SAR (Search And Rescue) antenna in Fig. 4 (right).
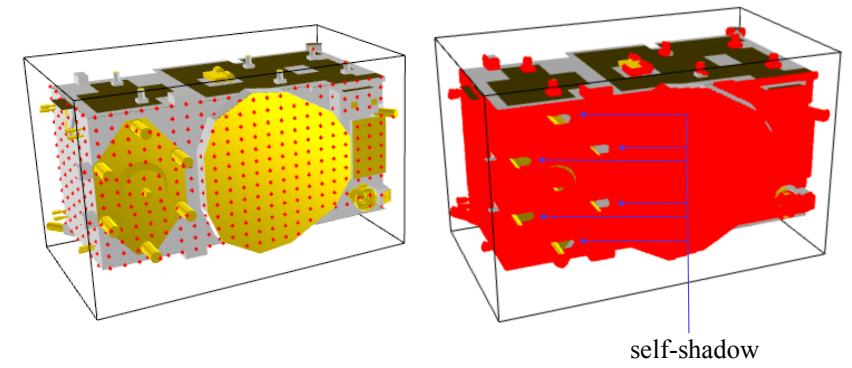

Figure 4: 3D visualisation of the results of intersection tests on the Galileo IOV bus model with pixel array spacing at $1 \mathrm{dm}$ (left) and $1 \mathrm{~cm}$ (right). Note that on the right figure, the shadows cast by the cylinders of the SAR antenna can be clearly seen.

\subsection{Testing the absolute accuracy of the algorithm}

Two test objects, a sphere and a planar surface, are used to assess the absolute accuracy of the proposed technique. For these objects, it is possible to evaluate the accelerations using analytical methods. Therefore, it is possible to use the analytical results as a baseline for evaluating the accuracy of the proposed, computational approach. In these tests, the pixel array spacing is set at $1 \mathrm{~mm}$ and the mass of the test objects are set at $1 \mathrm{~kg}$. Other key parameters in these tests are summarised in Table 3.

For both objects, the pixel array moves in the YOZ plane, scanning the objects from the $+y$ axis $(\theta=0)$ to the $-y$ axis $(\theta=180)$, in 1-degree increments, as shown in Fig. 5, where $\theta$ is the angle between the anti-ray direction and the $+y$ axis. With this scanning strategy, the $\mathrm{x}$ component of the incoming rays are always equal to zero, while the $y$ and $\mathrm{z}$ components are symmetric about the XZ plane.

Table 3: The key parameters for setting up the absolute accuracy test.

\begin{tabular}{cc}
\hline Items & Values \\
\hline solar constant & $1368 \mathrm{~W} \cdot \mathrm{m}^{-2}$ \\
reflectivity & 0.7 \\
specularity & 0.4 \\
resolution & $1 \mathrm{~mm}$ \\
area of the plane & $4.0 \mathrm{~m}^{2}$ \\
radius of the sphere & $1.0 \mathrm{~m}$ \\
mass & $1.0 \mathrm{~kg}$ \\
\hline
\end{tabular}

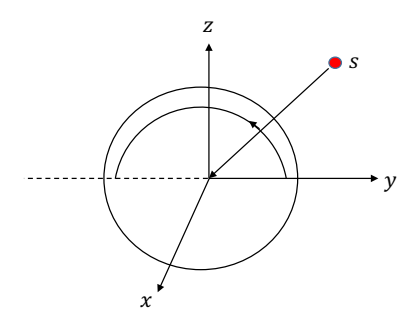

(a) sphere test

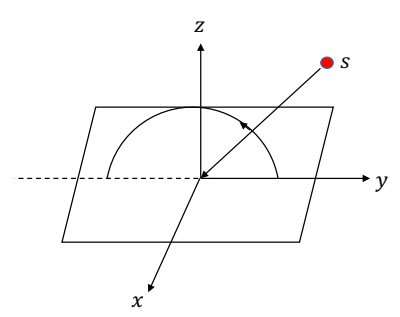

(b) plane test
Figure 5: The test cases for the sphere and plane, the sun is scanning from the $+\mathrm{y}$ to $-\mathrm{y}$.

\section{Plane test}

For the plane test, equation (5) is used as the analytical formula to calculate the solar radiation pressure. Figure 6 shows the acceleration for the plane test. The difference in the magnitude of the acceleration between the ray tracer and the analytical solution is shown in Fig. 7. The average value of the difference is $0.14 \mathrm{~nm} / \mathrm{s}^{2}$ and the standard deviation is $3.19 \mathrm{~nm} / \mathrm{s}^{2}$. 


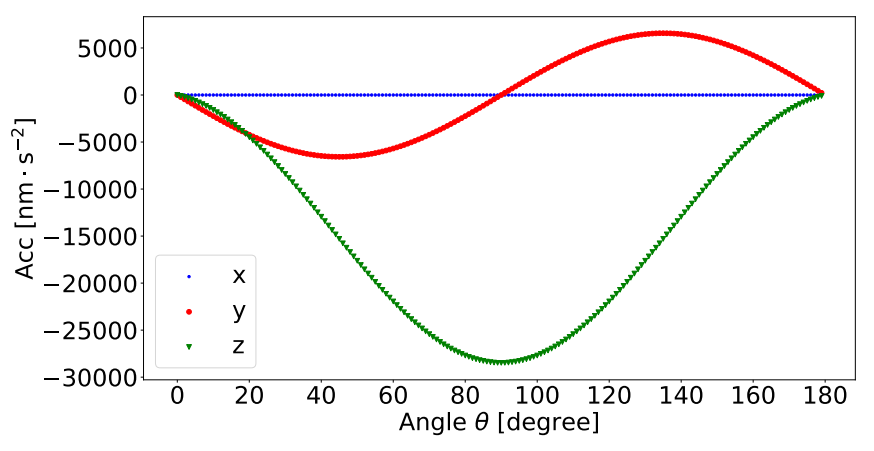

Figure 6: The force of the plane test

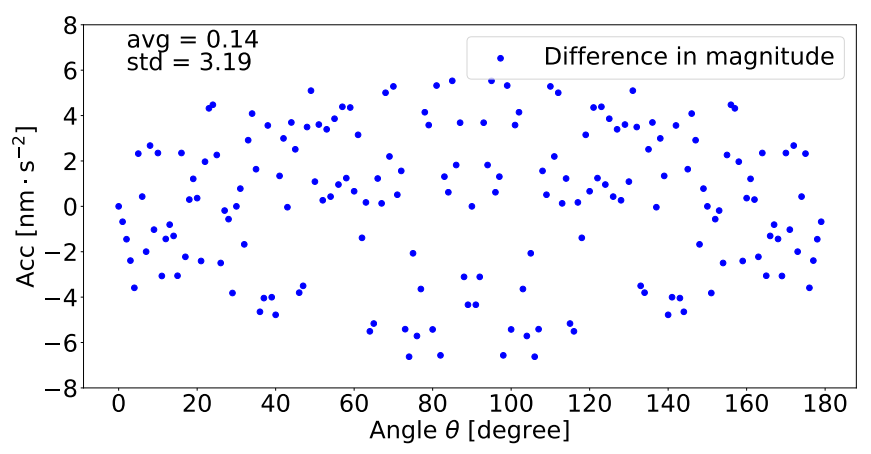

Figure 7: The difference between the ray tracer and analytical solution for the plane

\section{Sphere test}

As for the sphere, equation (5) is applied to the differential area and is integrated around the hemi-sphere. The integration gives out the analytical formula of the SRP force for spheres

$$
\mathbf{F}=\frac{W \pi R^{2}}{c}\left(1+\frac{4}{9} v-\frac{4}{9} \nu \mu\right) \mathbf{s}
$$

Figure 8 shows the acceleration from the ray tracer in the sphere test and the difference in magnitude between ray tracer and the analytical solution is shown in Fig. 9. The average value of the difference is $-0.046 \mathrm{~nm} / \mathrm{s}^{2}$ and the standard deviation is 0.112 $\mathrm{nm} / \mathrm{s}^{2}$.

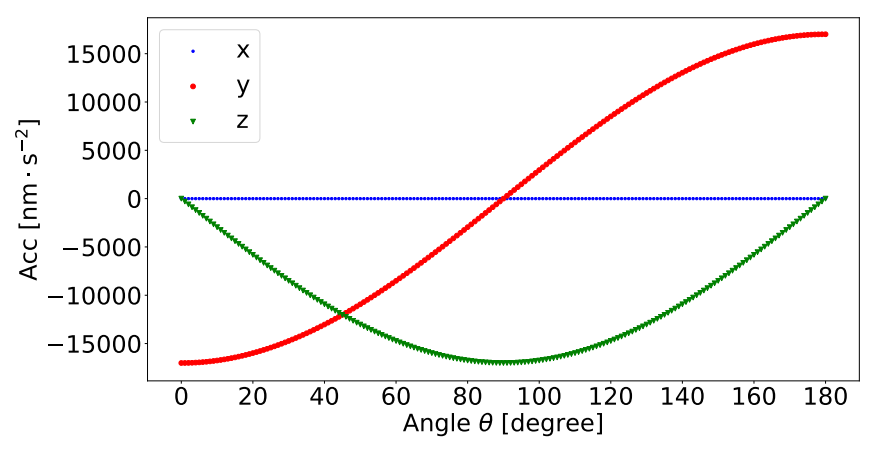

Figure 8: The acceleration from the sphere test

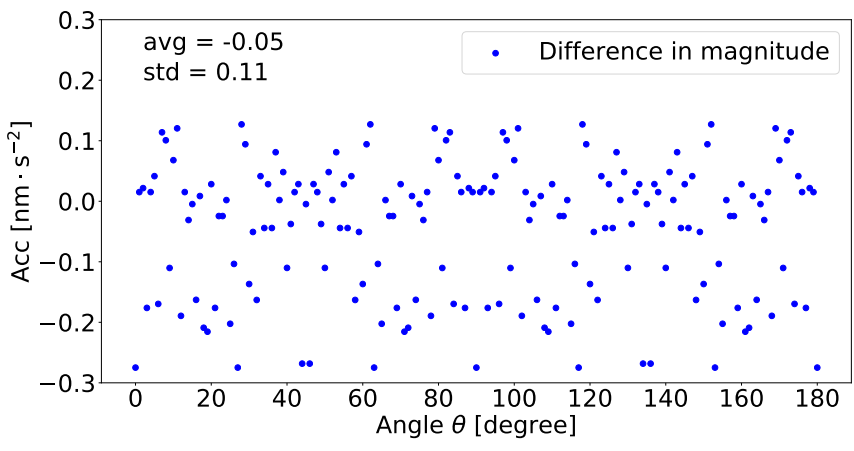

Figure 9: The difference between the ray tracer and analytical solution for the sphere

Note that the standard deviation of the differences is about 30 times smaller in the sphere test than it is in the planar surface test. This is because of errors in the pixel-array based technique that occur at the edges of the geometry. For instance, in the case of the sphere, which has a radius of $1 \mathrm{~m}$ in this test, the edge of the sphere is a circle of circumference $2 \pi \mathrm{m}$, and as the radiation source (simulated by the pixel array) pans across the sphere, this is nearly constant. On the other hand, for the planar surface, the perimeter of the edge of the geometry varies as the pixel array pans across the $\theta=0-180$ range. Therefore, when the radiation source is lower, as the pixel array is projected onto the cross-section of the planar surface, the perimeter/area ratio increases, and this increases the relative error at the edges. This link between the the linear dimensions of the pixel array grid cells and the magnitude of the errors in the ray-tracing technique are explored further in the next section.

\subsection{The effects of the pixel array resolution}

This is to test the effects of different sizes of the pixel in the ray tracing. In general, smaller pixel size will lead to higher modelling accuracy. In this test, 4 different resolutions including $1 \mathrm{dm}, 1 \mathrm{~cm}, 1 \mathrm{~mm}$ and $0.1 \mathrm{~mm}$ are chosen. We take $0.1 \mathrm{~mm}$ resolution as the baseline, the difference in force are calculated between the baseline and other resolutions. The GPS IIR and Galileo IOV satellites are tested in the yaw steering attitude (Montenbruck et al, 2015a) while Sentinel-1 satellite is tested in the roll steering attitude (Peter et al, 2017). The magnitude of the acceleration are shown in Fig. 10 for the bus of GPS IIR, Galileo IOV and the whole spacecraft of Sentinel-1.

Figure 11, Fig. 12 and Fig. 13 show the difference in acceleration for $1 \mathrm{dm}, 1 \mathrm{~cm}$ and $1 \mathrm{~mm}$ pixel resolutions for GPS IIR satellite, Galileo IOV satellite and Sentinel-1 separately. The error statistics on the three different resolutions are listed in Table 4. One interesting phenomenon is that the average value for GPS IIR with $1 \mathrm{~cm}$ resolution is biased. This is because of the antennas on the GPS IIR satellite bus. The diameter of those cylindrical antennas is around $1 \mathrm{~cm}$ which means the ray tracer may miss most of the antennas with $1 \mathrm{~cm}$ resolution. But these geometries can be captured by resolution of $1 \mathrm{~mm}$ and $0.1 \mathrm{~mm}$. Some experience can be learnt from these calculation results. If there are large number of components that have similar sizes of 
the pixel resolution, the pixel resolution for the ray tracer should be smaller in order to capture all the geometrical information.

The relative accuracy for different pixel spacing can be calculated by dividing the standard deviation by the average acceleration. The average accelerations for GPS IIR bus, Galileo IOV bus and the Sentinel- 1 spacecraft are $20.576 \mathrm{~nm} \cdot \mathrm{s}^{-2}, 28.110$ $\mathrm{nm} \cdot \mathrm{s}^{-2}$, and $81.381 \mathrm{~nm} \cdot \mathrm{s}^{-2}$ as shown in Fig. 10. The relative accuracy for different pixel resolutions is shown in Table 4. The relative accuracy is mainly driven by the pixel spacing, the relative accuracy of $1 \mathrm{dm}$ pixel spacing is about $1.7 \%$ and it gets almost 10 times smaller for $1 \mathrm{~cm}$ and so forth for $1 \mathrm{~mm}$ spacing. This is because as the pixel spacing gets smaller, the sampling error for the cross-section area gets smaller and the error in the cross-section has linear impact to the SRP acceleration (See equation 5). For most of the satellites, a resolution of $1 \mathrm{~mm}$ should be good enough for the SRP modelling. Thus in the following multiple reflection test, the spacing of the pixel array is set to be $1 \mathrm{~mm}$.

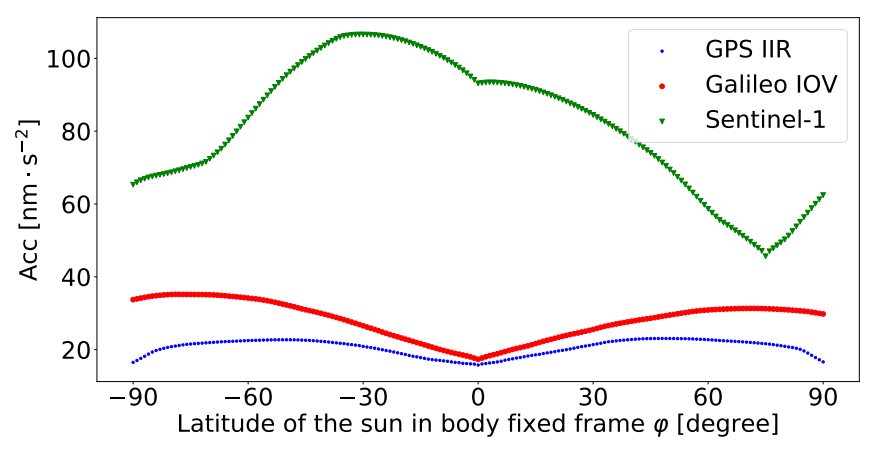

Figure 10: The errors with $1 \mathrm{~mm}$ pixel resolution

\begin{tabular}{cccc}
\multicolumn{4}{c}{ Table 4: The error statistics of different resolutions [unit:nm $\cdot \mathrm{s}^{-2}$ ] } \\
\hline Resolution & $1 \mathrm{dm}$ & $1 \mathrm{~cm}$ & $1 \mathrm{~mm}$ \\
\hline Galileo IOV Avg. & $-0.711 \mathrm{E}-2$ & $-0.815 \mathrm{E}-3$ & $3.706 \mathrm{E}-5$ \\
GPS IIR Avg. & -0.273 & -0.091 & $0.755 \mathrm{E}-3$ \\
Sentinel-1 Avg. & -0.281 & 0.032 & $-0.641 \mathrm{E}-3$ \\
Galileo IOV Std. & 0.495 & 0.039 & 0.004 \\
GPS IIR Std. & 0.335 & 0.038 & 0.002 \\
Sentinel-1 Std. & 1.416 & 0.139 & 0.013 \\
Galileo IOV rel. & $1.761 \%$ & $0.139 \%$ & $0.014 \%$ \\
GPS IIR rel. & $1.628 \%$ & $0.185 \%$ & $0.010 \%$ \\
Sentinel-1 rel. & $1.739 \%$ & $0.171 \%$ & $0.016 \%$ \\
\hline
\end{tabular}

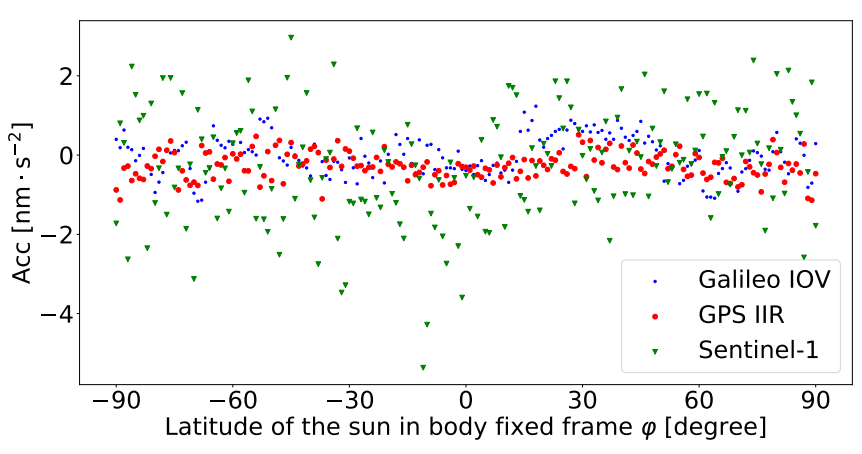

Figure 11: The errors with $1 \mathrm{dm}$ pixel resolution

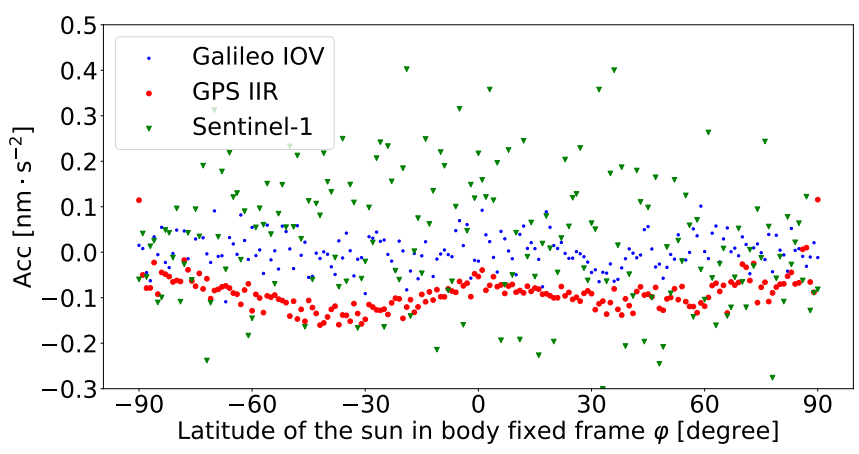

Figure 12: The errors with $1 \mathrm{~cm}$ pixel resolution

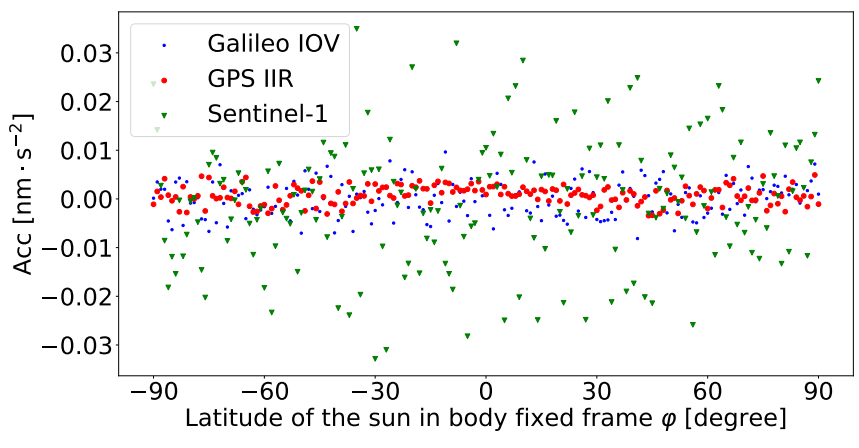

Figure 13: The errors with $1 \mathrm{~mm}$ pixel resolution

\subsection{Speedup test}

In order to do the speeding up test, different resolutions of the pixel array are applied in this test on a personal computer. The results are compared between UCL ( University College London ) SRP software ( denoted as "UCL raw"), the ray tracing without BVH ( denoted as "no BVH") and the ray tracing with BVH ( denoted as "with BVH" ). We take one Galileo IOV satellite bus and one GPSIIR satellite bus as test objects. Assuming the spacecraft is under nominal attitude, the longitude of the sun is fixed to 180 degrees while latitude varies from -90 to 90 degrees. Table 5 and Table 6 show the average time consumption of 180 different directions. The approach with BVH 
algorithm is about $30-50$ times faster than the UCL raw software.

Table 5: The speedup test for a GPS IIR satellite bus

\begin{tabular}{lccc}
\hline Method & UCL raw & no BVH & with BVH \\
\hline $1 \mathrm{dm}(\mathrm{ms})$ & 106.78 & 53.52 & 2.97 \\
$1 \mathrm{~cm}(\mathrm{~s})$ & 9.27 & 5.38 & 0.32 \\
$1 \mathrm{~mm}$ (min) & 14.45 & 8.79 & 0.46 \\
avg. speedup & 1.0 & 1.78 & 32.69 \\
\hline
\end{tabular}

Table 6: The speedup test for one Galileo IOV satellite bus

\begin{tabular}{lccc}
\hline Method & UCL raw & no BVH & with BVH \\
\hline $1 \mathrm{dm}(\mathrm{ms})$ & 75.601 & 35.45 & 1.11 \\
$1 \mathrm{~cm}(\mathrm{~s})$ & 5.30 & 3.30 & 0.10 \\
$1 \mathrm{~mm}(\mathrm{~min})$ & 8.09 & 8.79 & 0.15 \\
avg. speedup & 1.0 & 1.75 & 59.39 \\
\hline
\end{tabular}

\subsection{Multiple reflection test}

In the solar radiation pressure modelling for GLONASS satellites, the secondary reflection effects in the force can cause 0.1 $\mathrm{m}$ error in the along-tack after 12 hours (Ziebart, 2001). The 1 st reflection is defined as the the ray hit and bounce off but not any further intersections. Thus the secondary reflection effect is the error due to omitting 2 nd, 3rd etc. intersections. In this study, we take the 10-reflection as a base line, the effects of multiple reflections are investigated in two aspects, one is the proportion of intersection number for multiple reflections over the base line, the other is the difference in acceleration between multiple reflections and the base line.

Figure 14, Fig. 15, and Fig. 16 show the proportion of intersection number with respect to the reflection number for GPS IIR, Galileo IOV and Sentinel-1 satellites respectively. As Fig. 17 and Table 7 show, the primary rays can only capture about $85 \%, 91 \%$, and $84 \%$ of the total rays for GPS IIR, Galileo IOV satellite bus and Sentinel-1 separately. The secondary reflection can capture over $96 \%$ while the third reflection can capture over $99 \%$ for GPS IIR and Galileo IOV satellite bus. Because of the complex shape of Sentinel-1, even the third reflection can just capture around $91 \%$. However, this does not mean the accuracy in the acceleration. The reduction of the energy of the reflected rays make the contribution of multiple reflected rays to the acceleration gets smaller and smaller.

The multiple reflection effects of Sentinel-1 spacecraft would be obvious due to its large SAR (Synthetic Aperture Radar) antenna and the big solar panels, see Fig. 3. The difference in acceleration for GPS IIR (bus only), Galileo IOV (bus only) and Sentinel1 (whole spacecraft) between the 1st, 2nd, 3rd reflection and the base line are shown in Fig. 18, Fig. 19, Fig. 20. In terms of the percentage in acceleration magnitude due to multiple reflections, Galileo IOV satellite bus gets the largest (around 1\%) among the three space crafts due to its large area to mass ratio. Figure 21 shows the error percentage of acceleration magnitude for 1 st, 2nd and 3rd reflections.

The errors in acceleration due to the 1 st reflection can reach to $0.2 \mathrm{~nm} / \mathrm{s}^{2}$ and $0.4 \mathrm{~nm} / \mathrm{s}^{2}$ for Galileo IOV and Sentinel-1
Table 7: This table shows the average proportion of intersections w.r.t reflection number

\begin{tabular}{cccc}
\hline \# of reflection & GPS IIR & Galileo IOV & Sentinel-1 \\
\hline 1 & $84.988 \%$ & $91.221 \%$ & $84.683 \%$ \\
2 & $96.935 \%$ & $98.123 \%$ & $89.291 \%$ \\
3 & $99.127 \%$ & $99.137 \%$ & $91.341 \%$ \\
4 & $99.574 \%$ & $99.534 \%$ & $92.941 \%$ \\
5 & $99.755 \%$ & $99.730 \%$ & $94.321 \%$ \\
6 & $99.828 \%$ & $99.842 \%$ & $95.586 \%$ \\
7 & $99.897 \%$ & $99.919 \%$ & $96.772 \%$ \\
8 & $99.915 \%$ & $99.963 \%$ & $97.898 \%$ \\
9 & $99.940 \%$ & $99.984 \%$ & $98.971 \%$ \\
10 & $100 \%$ & $100 \%$ & $100 \%$ \\
\hline
\end{tabular}

separately. For the 2nd and 3rd reflections, the errors get 2 or 3 order of magnitude smaller. In terms of the percentage error in acceleration magnitude, the percentage can reach $1 \%, 0.1 \%$ and $0.035 \%$ for 1 st, 2 nd and 3 rd reflections.

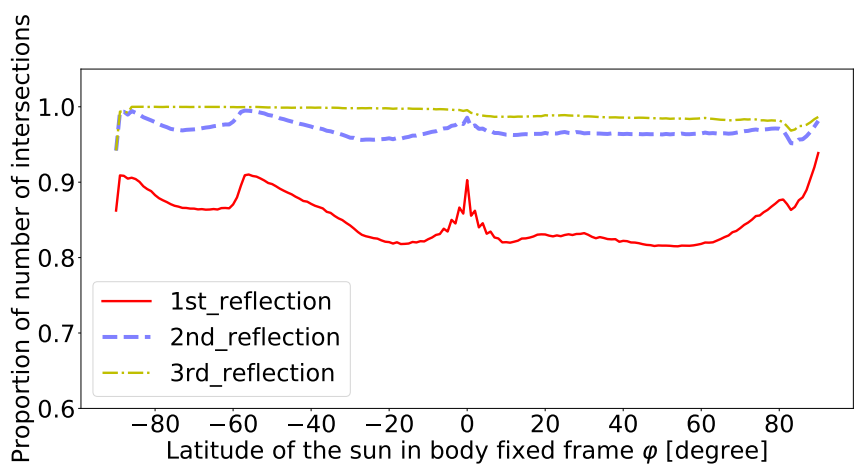

Figure 14: The difference in the proportion of number of intersection between different number of reflection for GPS IIR satellite bus

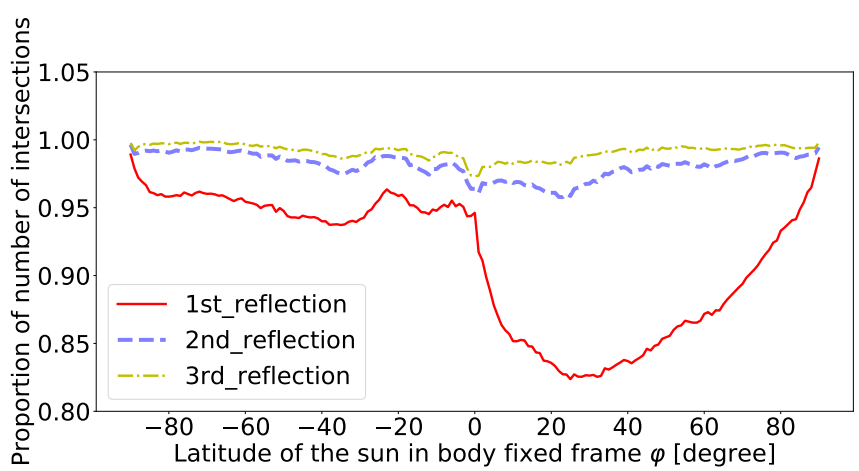

Figure 15: The difference in the proportion of number of intersection between different number of reflection for Galileo IOV satellite bus

The impact of multiple reflection is relative weak comparing with the errors in the knowledge of optical properties, the operational mass of the spacecraft and the accuracy of the spacecraft geometry model. The ray tracing approach for SRP modelling is required to be in $\mathrm{sub}-\mathrm{nm} / \mathrm{s}^{2}$ level (Montenbruck et al, $2015 \mathrm{~b}$ ), thus the multiple reflection impact should be considered. The inputs ( i.e. the TSI, the optical properties, the ge- 


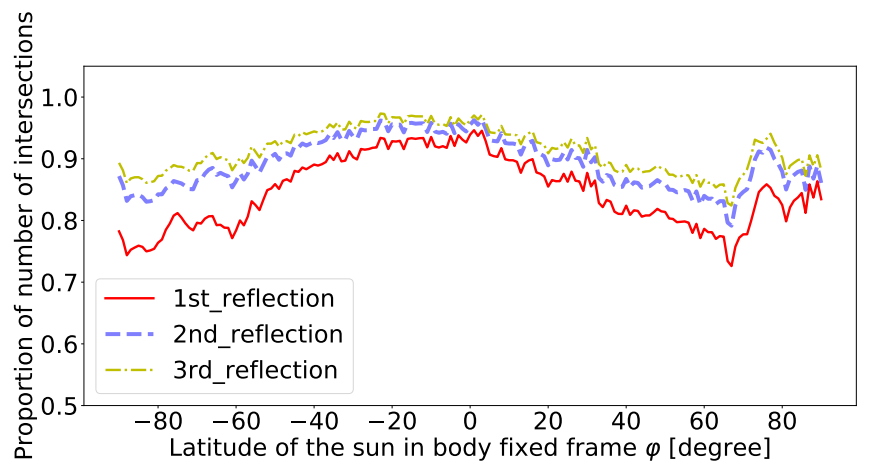

Figure 16: The difference in the proportion of number of intersection between different number of reflection for Sentinel-1 spacecraft

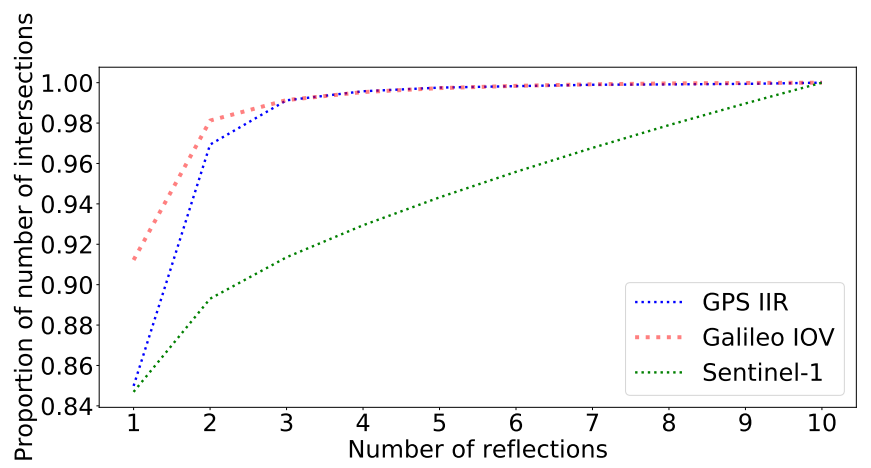

Figure 17: The average proportion of number of intersection for different number of reflection

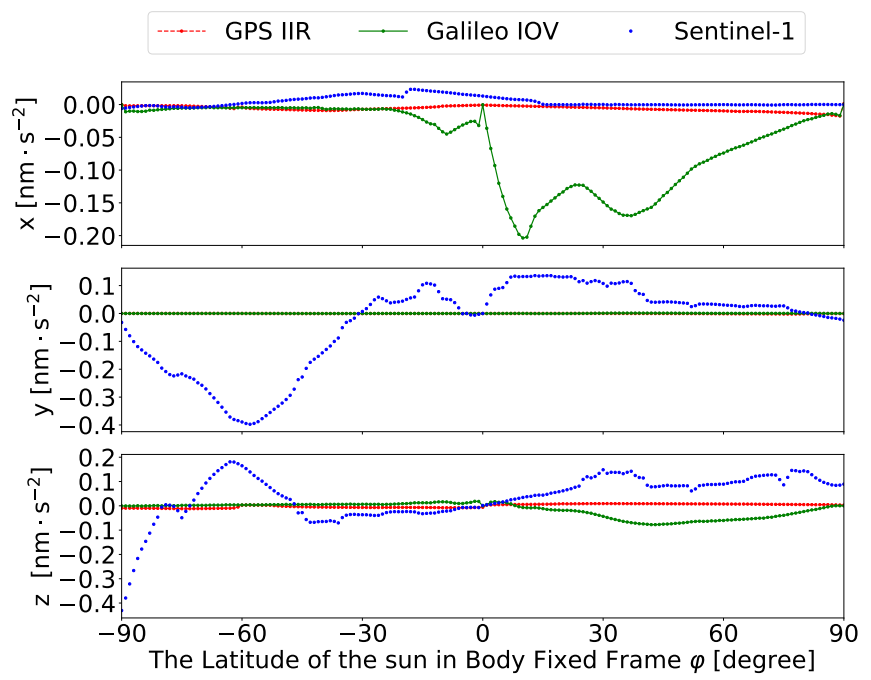

Figure 18: The errors in acceleration for the 1st reflection
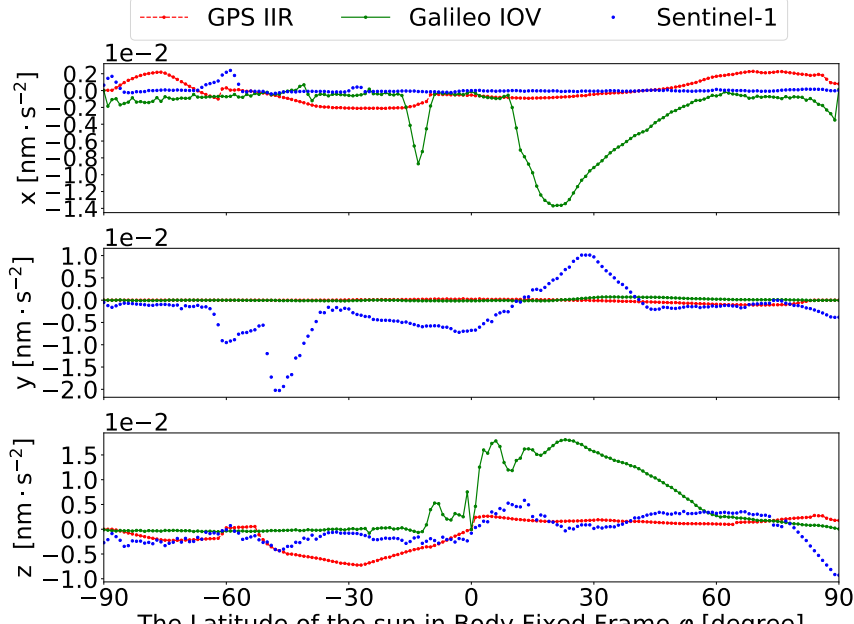

The Latitude of the sun in Body Fixed Frame $\varphi$ [degree]

Figure 19: The errors in acceleration for the 2nd reflection

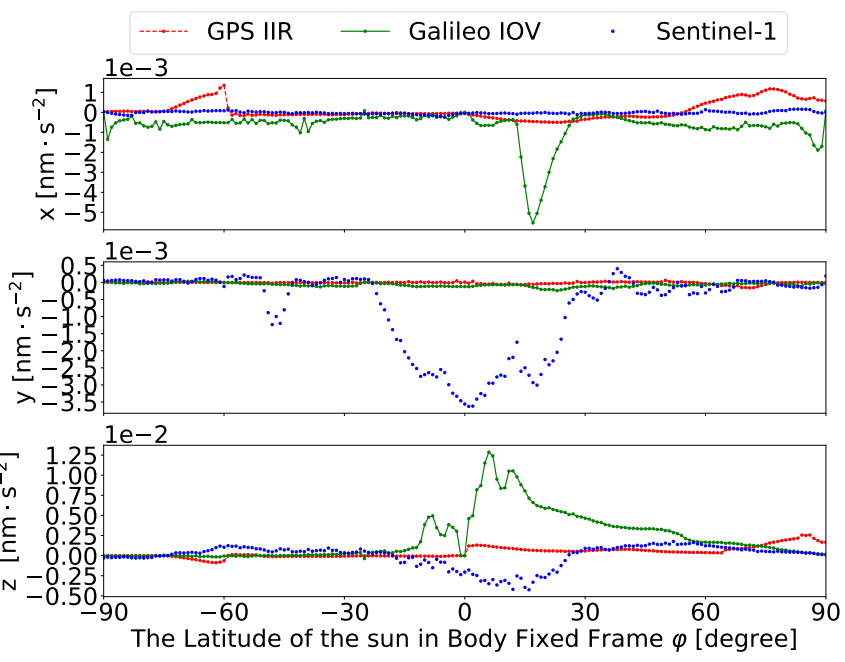

Figure 20: The errors in acceleration for the 3rd reflection 


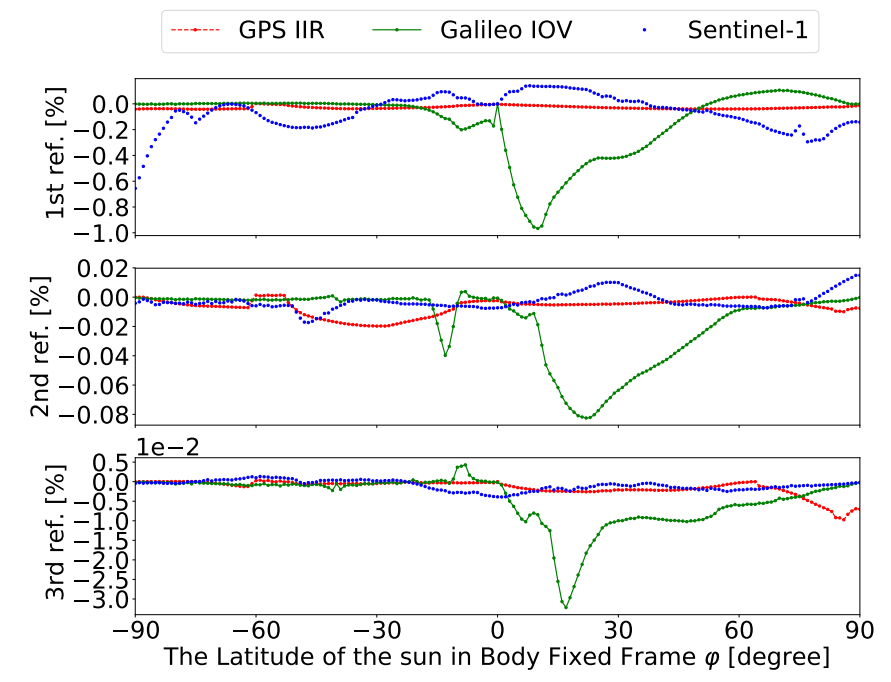

Figure 21: The percentage of errors due to 1st, 2nd and 3rd reflections for GPS IIR, Galileo IOV and Sentinel-1 spacecraft

ometrical model and the satellite mass ) to the ray tracing approach are encouraged to be made more accurate.

\section{Conclusion}

This research starts from the physical basis of radiation force then a ray tracing based high-fidelity radiation pressure modelling technique is followed. Thereafter, the BVH data structure is applied to the ray tracing process and it makes the ray tracing process run faster. The tests focus on 4 aspects including the accuracy of the ray tracing technique for radiation pressure modelling, the speed up efficiency of the $\mathrm{BVH}$ on radiation pressure modelling, the effects of multiple reflections, the effects of the pixel resolution.

The accuracy tests on a plane and a sphere show that this ray tracing technique can reach an accuracy of about several nano meter per square seconds with a pixel array resolution of $1 \mathrm{~mm}$. In practice, this accuracy could be affected by the relative ratio of the actual size of the spacecraft over the pixel array resolution. In addition, in the test of the pixel array resolution for GPS IIR spacecraft, a bias has been found for the resolution of $1 \mathrm{dm}$ and $1 \mathrm{~cm}$. The reason for this is that there are many cylindrical antennas that have a radius of around $1 \mathrm{~cm}$. A resolution of $1 \mathrm{~cm}$ in the pixel array may cause a large sum of rays to miss those components. The effects can be seen clearly in Fig. 12. In order to balance between the accuracy and the computation time, a proper resolution of the pixel array has to be chosen according to the details of the spacecraft.

The test results on multiple reflections for GPS IIR and Galileo IOV satellites show that the first three reflections for each ray can capture over $99 \%$ of the total intersections. Though the first three reflections can only capture around $90 \%$ of the total intersections for Sentinel-1, the contribution in acceleration due to multiple reflections gets smaller and smaller as the increase of the reflection number due to the reduced energy of the reflected rays. The tests on the effects of multiple reflections in acceleration show that the secondary intersection effect is nonnegligible for both Galileo IOV satellite and Sentinel-1 spacecraft. The magnitude of acceleration due to secondary intersection can reach $0.2 \mathrm{~nm} / \mathrm{s}^{2}$ and $0.4 \mathrm{~nm} / \mathrm{s}^{2}$ for Galileo IOV and Sentinel-1 spacecraft separately. Considering the 3rd intersection can make the error percentage less than $0.035 \%$. Thus in the modelling of solar radiation pressure with ray tracing technique, the 3 rd reflection should be considered. It is the first time to do systematic and quantitative study on the secondary and higher order intersection effect and it shows conclusively the effect is non-negligible for certain classes of mission.

Despite the ray tracing approach itself can reach high accuracy in SRP modelling, the accuracy is limited by the inputs to the approach. Such as the variation of the TSI, the optical properties, the geometrical model. The current input data for high precision SRP modelling is not satisfied to some extent. Therefore, the measurements of the surface optical properties of the materials and the operational mass of the satellite are encouraged to be make public.

\section{Acknowledgements}

This work is jointly funded by University College London and China Scholarship Council. Thanks for collegue who worked on the UCL (University College London) Solar Radiation Pressure Modelling software in the past and present.

\section{References}

Arnold D, Meindl M, Beutler G, Dach R, Schaer S, Lutz S, Prange L, Sośnica K, Mervart L, Jäggi A (2015) CODE's new solar radiation pressure model for GNSS orbit determination. Journal of Geodesy 89(8):775-791, DOI 10.1007/s00190-015-0814-4

Bittner J, Hapala M, Havran V (2015) Incremental BVH construction for ray tracing. Computers and Graphics (Pergamon) 47:135-144, DOI 10.1016/j. cag.2014.12.001

Casotto S, Gini F, Panzetta F, Bardella M (2013) Fully dynamic approach for GOCE precise orbit determination. Bollettino di Geofisica Teorica ed Applicata 54(4):367-384, DOI 10.4430/bgta0108

Cerri L, Berthias JP, Bertiger WI, Haines BJ, Lemoine FG, Mercier F, Ries JC, Willis P, Zelensky NP, Ziebart M (2010) Precision orbit determination standards for the Jason series of altimeter missions. Marine Geodesy 33(SUPPL. 1):379-418, DOI 10.1080/01490419.2010.488966

European Global Navigation Satellite Systems Agency (2017) Galileo Satellite Metadata. URL https://www.gsc-europa.eu/ galileo-gsc-overview/system

Fliegel HF, Gallini TE, Swift ER (1992) Global Positioning System Radiation Force Model for geodetic applications. Journal of Geophysical Research 97(B1):559, DOI 10.1029/91JB02564

Gao F, Peng B, Zhang Y, Evariste NH, Liu J, Wang X, Zhong M, Lin M, Wang $\mathrm{N}$, Chen R, Xu H (2015) Analysis of HY2A precise orbit determination using DORIS. Advances in Space Research 55(5):1394-1404, DOI 10.1016/ j.asr.2014.11.032

Grey S, Marchand R, Ziebart M, Omar R (2017) Sunlight Illumination Models for Spacecraft Surface Charging. IEEE Transactions on Plasma Science 45(8):1898-1905, DOI 10.1109/TPS.2017.2703984

Günther J, Popov S, Seidel HP, Slusallek P (2007) Realtime ray tracing on GPU with BVH-based packet traversal. In: IEEE/ EG Symposium on Interactive Ray Tracing 2007 Proceedings, IRT, pp 113-118, DOI 10.1109/RT.2007. 4342598

Hastings D, Garrett H (2004) Spacecraft-Environment Interactions (Cambridge Atmospheric and Space Science Series). Cambridge University Press, Cambridge, DOI doi:10.1017/CBO9780511525032 
Hormann K, Agathos A (2001) The point in polygon problem for arbitrary polygons. Computational Geometry: Theory and Applications 20(3):131-144, DOI 10.1016/S0925-7721(01)00012-8

Kang Z, Tapley B, Bettadpur S, Ries J, Nagel P, Pastor R (2006) Precise orbit determination for the GRACE mission using only GPS data. Journal of Geodesy 80(6):322-331, DOI 10.1007/s00190-006-0073-5

Lauterbach C, Garland M, Sengupta S, Luebke D, Manocha D (2009) Fast BVH Construction on GPUs. Computer Graphics Forum 28(2):375-384, DOI 10.1111/j.1467-8659.2009.01377.x

Lebedev PN (1901) Experimental Examination of Light Pressure. Annalen der Physik 6(433):1-26, DOI 10.1002/andp.19013111102

Meindl M, Beutler G, Thaller D, Dach R, Jaggi A (2013) Geocenter coordinates estimated from GNSS data as viewed by perturbation theory. Advances in Space Research 51(7):1047-1064, DOI 10.1016/j.asr.2012.10.026

Montenbruck O, Gill E (2005) Satellite Orbits Models, Methods and Applications, corrected edn. Springer Berlin Heidelberg, Berlin, Heidelberg, DOI 10.1007/978-3-642-58351-3, URL http://link.springer.com/ 10.1007/978-3-642-58351-3

Montenbruck O, Schmid R, Mercier F, Steigenberger P, Noll C, Fatkulin R, Kogure S, Ganeshan AS (2015a) GNSS satellite geometry and attitude models. Advances in Space Research 56(6):1015-1029, DOI 10.1016/j.asr.2015. 06.019, URL http: //dx.doi.org/10.1016/j.asr.2015.06.019

Montenbruck O, Steigenberger P, Hugentobler U (2015b) Enhanced solar radiation pressure modeling for Galileo satellites. Journal of Geodesy 89(3):283297, DOI 10.1007/s00190-014-0774-0

Nichols E, Hull G (1902) Pressure due to light and heat radiation. The Astrophysical Journal 15:62

Nichols EF, Hull GF (1903) The Pressure Due to Radiation. Proceedings of the American Academy of Arts and Sciences 38(20):559, DOI 10.2307/ 20021808

Peter H, Jäggi A, Fernández J, Escobar D, Ayuga F, Arnold D, Wermuth M, Hackel S, Otten M, Simons W, Visser P, Hugentobler U, Féménias P (2017) Sentinel-1A First precise orbit determination results. Advances in Space Research 60(5):879-892, DOI 10.1016/j.asr.2017.05.034

Sibthorpe A, Bertiger W, Desai SD, Haines B, Harvey N, Weiss JP (2011) An evaluation of solar radiation pressure strategies for the GPS constellation. Journal of Geodesy 85(8):505-517, DOI 10.1007/s00190-011-0450-6

Sibthorpe AJ (2006) Precision Non-Conservative Force Modelling For Low Earth Orbiting Spacecraft. PhD thesis, University College London

Solano CJR (2014) Impact of non-conservative force modeling on GNSS satellite orbits and global solutions. PhD thesis, Technische Universität München

Sopin D, Bogolepov D, Ulyanov D (2011) Real-time SAH BVH construction for ray tracing dynamic scenes. Proceedings of the 21 th International Conference on Computer Graphics and Vision (GraphiCon) pp 74-77

Springer TA, Beutler G, Rothacher M (1999) A New Solar Radiation Pressure Model for GPS Satellites. GPS Solutions 2(3):50-62, DOI 10.1007/ PL00012757

Tan B, Yuan Y, Zhang B, Hsu HZ, Ou J (2016) A new analytical solar radiation pressure model for current BeiDou satellites: IGGBSPM. Scientific Reports 6:1-9, DOI 10.1038/srep32967

Williams A, Barrus S, Keith R, Shirley MP (2003) An efficient and robust raybox intersection algorithm. Journal of Graphics Tools 10:54, DOI 10.1080/ 2151237X.2005.10129188

Ziebart M (2001) High Precision Analytical Solar Radiation Pressure Modelling for GNSS Spacecraft. PhD thesis, The University of East London

Ziebart M (2004) Generalised Analytical Solar Radiation Pressure Modelling Algorithm for Spacecraft of Complex Shape. Journal of Spacecraft and Rockets 41(5):840-848

Ziebart M, Adhya S, Sibthorpe A, Cross P (2003) GPS Block IIR NonConservative Force Modelling : Computation and Implications. In: ION GPS/GNSS, September, pp 2671-2678

Ziebart M, Adhya S, Sibthorpe A, Edwards S, Cross P (2005) Combined radiation pressure and thermal modelling of complex satellites: Algorithms and on-orbit tests. Advances in Space Research 36(3):424-430, DOI 10.1016/j.asr.2005.01.014 